\title{
Effects of game-based balance training with constraint-induced movement therapy on lower extremity function and balance confidence levels in women with total knee replacement
}

\author{
Ho-Suk Choi, Won-Seob Shin \\ Department of Physical Therapy, College of Health and Medical Science, Daejeon University, Daejeon, Republic of Korea
}

Objective: Degenerative knee arthritis is the most common disease that occurs in older people. Constriction-induced movement therapy (CIMT) has been reported to be as an effective treatment for the impairments, such as asymmetric weight-bearing and reduced balance that occurs after receiving a total knee replacement (TKR). Game-based rehabilitation training for persons with TKR is interesting and provides a variety of feedback.

Design: Randomized controlled trial.

Methods: Thirty-six subjects with TKR were randomly assigned to either the CIMT game training $(\mathrm{n}=12)$, general game training $(n=12)$, or the control $(n=12)$ group. Each group underwent twelve sessions (30 min/d, $3 \mathrm{~d} / \mathrm{wk}$ for 4 weeks). In the CIMT game training group, the application of CIMT adjusted the weight of the pressure delivered from the two boards used in Wii games. In the general game training group, the game was played without adjusting the weight of pressure. The game training used the Wii Fit's Ski Slalom application. Subjects were assessed with the Western Ontario and McMaster Universities Osteoarthritis Index (WOMAC) and the Activities-specific Balance Confidence (ABC) Scale.

Results: All three groups showed significant improvements in pain, stiffness and physical function, total WOMAC scores, and $\mathrm{ABC}$ scores after the intervention $(p<0.05)$. Significant differences were observed in physical function, total WOMAC scores, and $\mathrm{ABC}$ scores of the CIMT game training group compared with the other groups $(p<0.05)$.

Conclusions: The CIMT game training and general game training exhibited improvements on stiffness, but the CIMT game training exhibited a larger effect on lower extremity function and balance confidence levels.

Key Words: Arthritis, Arthroplasty, Exercise therapy, Knee

\section{Introduction}

Degenerative knee arthritis is one of the most common diseases in the elderly population [1]. The surgery that represents degenerative knee arthritis is knee arthroplasty, but there are still problems that exist after surgery. The patient experiences an asymmetrical movement pattern characterized by reduced knee extension strength of the operated side and limitation of the knee extension range of motion [2].
In addition, $20 \%$ of patients who undergo surgery still experience knee pain, resulting in asymmetrical weight bearing onto the non-operated leg [3]. As a result, pain, limited range of motion, and muscle weakness of the knee reduces the ability to maintain balance. In addition, the activity of the patient with reduced balance ability leads to fear of falling, which lowers the stability of their posture [4]. This leads to a relatively low level of confidence, which is a factor that affects physical function and activity and also changes the

Received: 27 February, 2019 Revised: 17 March, 2019 Accepted: 17 March, 2019

Corresponding author: Won-Seob Shin (ORCID https://orcid.org/0000-0002-6515-7020)

Department of Physical Therapy, College of Health and Medical Science, Daejeon University, 62 Daehak-ro, Dong-gu, Daejeon 34520, Republic of Korea Tel: 82-42-280-2294 Fax: 82-42-280-2295 E-mail: shinws@dju.kr

(c) This is an Open-Access article distributed under the terms of the Creative Commons Attribution Non-Commercial License (http://creativecommons.org/licenses/ by-nc/4.0) which permits unrestricted non-commercial use, distribution, and reproduction in any medium, provided the original work is properly cited.

Copyright (๑ 2019 Korean Academy of Physical Therapy Rehabilitation Science 
quality of life. Therefore, after the total knee replacement (TKR) arthroplasty, muscular strength, range of motion, and pain as well as balance abilities are important to address [5].

In recent years, previous studies have attempted to overcome the problems of various exercises and treatments using virtual reality and games [6]. There is a steady increase in game training studies for balance and cognition enhancement. One of the game systems is the Nintendo Wii and the motion control game using the Wii Balance Board (WBB). It is available for a wide range of ages and is popular and inexpensive. In addition, it has increased the participation and concentration of patients more than the existing rehabilitation environment, and it is being to complement an exercise environment focusing on balance [7]. Indeed, although the Nintendo Wii has shown potential to serve as a therapeutic tool in the rehabilitation of the elderly population and stroke survivors, few studies have been applied and have focused mainly on orthopedic patients. The WBB method for the anterior cruciate ligament reconstruction and the postoperative rehabilitation of the knee joint arthroplasty did not differ from the effects produced by conventional treatment on pain, range of motion and lower extremity function [8]. Therefore, this study was conducted to understand the therapeutic effect of the Nintendo Wii system when the exercise environment of the Nintendo Wii system was set for the general public. The existing game system was set up to be used by non-therapeutic target subjects and therefore, a treatment environment was made that could provide a greater amount of intensive stimulation to the rehabilitation of orthopedic patients.

Constraint-induced movement therapy (CIMT) further increases the use of the paralyzed side of the person affected by stroke. CIMT is one of the effective rehabilitation methods that forces the paralyzed side to move. Previous studies focused on improving the asymmetric weight-bearing of stroke survivors showed improvements in static and dynamic balance abilities of persons with stroke. There have been various interventions performed to improve the problems asymmetric weight-bearing and reduced balance in patients with a TKR [9-11]. The CIMT can serve to be more effective for weight-bear training, but studies using CIMT for TKR patients are rare.

Therefore, the purpose of this study was to investigate the effect of virtual reality-based game training using CIMT therapy on lower limb function, dynamic balance, and balance self-confidence of persons with knee arthroplasty on one leg.

\section{Methods}

\section{Participants}

The subjects included in this study were patients with a TKR on one leg who were admitted to Cheonan Medical Center in Cheonan. The selection criteria were one week after surgery and being able to walk without using walking aids. The exclusion criteria was an experience of two or more falls, other orthopedic problems in the lower limbs, severe sensory and cognitive impairment, nervous system or vestibular disorders, or surgery on other sites within 3 months.

The subjects were selected as those who had undergone knee arthroplasty for a week after receiving a knee arthritis diagnosis, those who did not have pain in the operative side of the lower extremity, and those who could respond correctly to the instructions. In addition, those who experienced two or more falls, and those with severe visual impairments were excluded from the study.

A pilot study was conducted to determine the sample size. The G* power (version 3.1.9.2; Heinrich-Heine-Universität, Düsseldorf, Germany) was used for alpha 0.05 , and the power of 0.8 was used for each sample. The size of the effect was 0.55 to 0.6 . The sample size was 10 , and a total of 36 subjects were selected considering the dropout rate of $20 \%$. A total of 36 subjects who agreed with the selection criteria were randomly assigned to the CIMT game training group $(n=12)$, the general game training group $(n=12)$, or the self-exercising group $(n=12)$. After being fully explained about the purpose and process of the study, all subjects decided to voluntarily participate in the experiment. Participants provided signed consent after receiving verbal and written information about the study. All the procedures and methods of this study have been approved by the Institutional Review Board of Daejeon University (IRB No. 1040647-201712-HR-005) and follow the principles outlined in the Declaration of Helsinki.

\section{Procedure}

This study was performed on 36 patients who underwent knee arthroplasty for degenerative knee arthritis. The CIMT game training group, general game training group, and the self-exercise group were randomly assigned to each group. The CIMT game training group and the general game training group performed game-based balance training three times a week for 30 minutes for 4 weeks. The self-exercising group received a printed sheet with the description of the ex- 


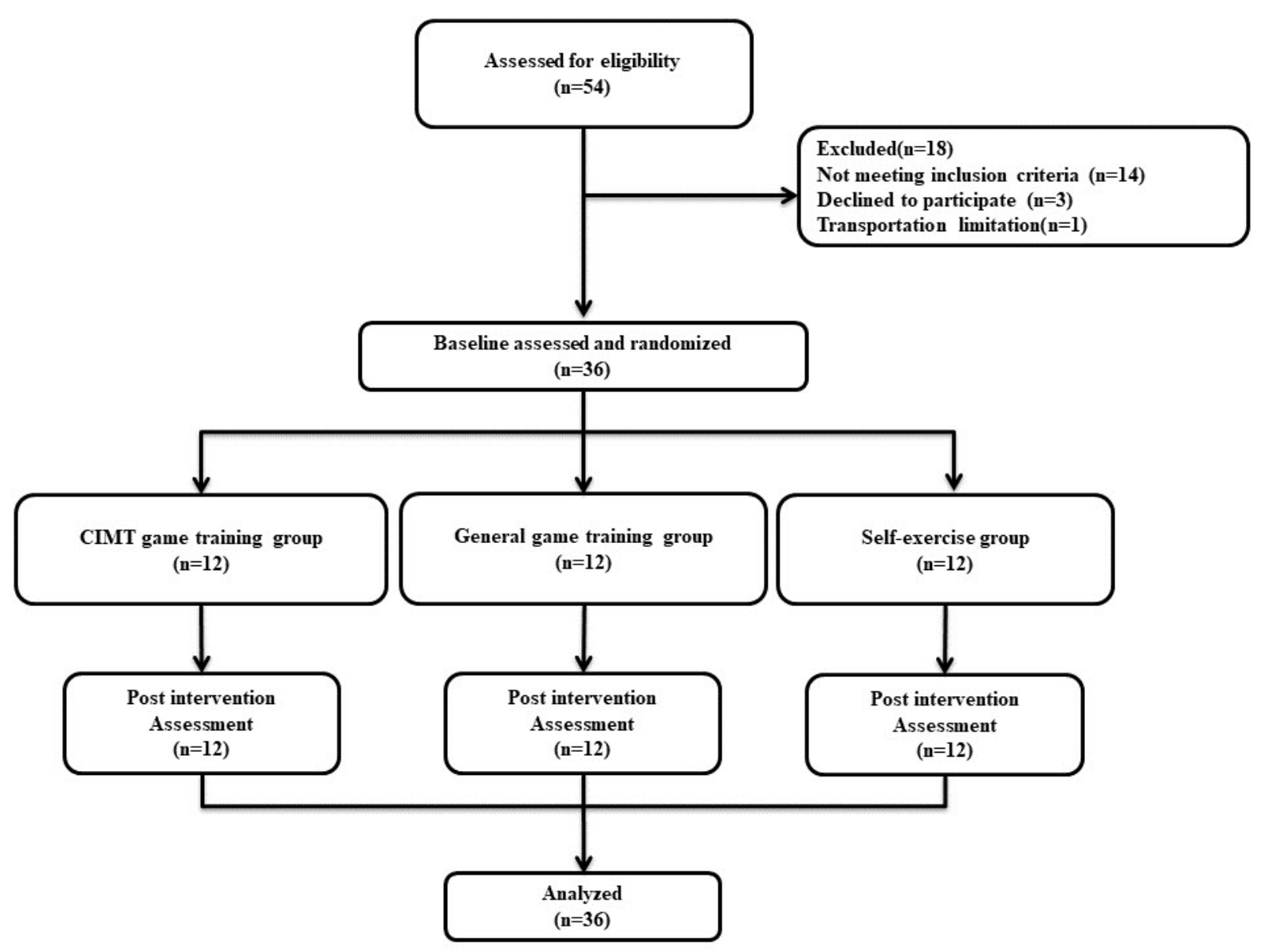

Figure 1. Flowchart of participants through the trial. CIMT: constraint-induced movement therapy.

ercises, and were recommended to perform the self-exercises three times a week for 30 minutes for 4 weeks. After the intervention, post-test was performed and each measured value was analyzed. The design of this study is as follows (Figure 1).

The CIMT game training group used the Ski Slalom application of the Nintendo's game system. When the subject moved their weight to the left or right, the character moved through the flags to the left or right (Figure 2).

The subjects were placed in the center of two WBBs, one foot on each board. One WBB had four sensors, located at the edges of each end to measure the weight being loaded. Thus, a total of eight signals measured in two WBBs were collected in the computer via Bluetooth. The signals collected from the computer were used to calculate the center of pressure (COP) of each limb using a Matlab program. The measured COP was calculated via the Arduino Duemilanove as a virtual $\mathrm{COP}$ according to the following equation and was sent to the third WBB.

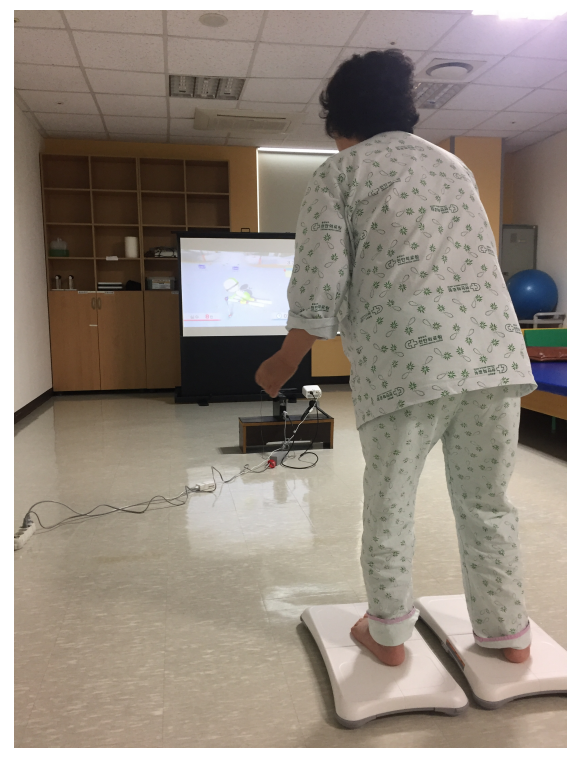

Figure 2. Game-based balance training with constraint-induced movement therapy. 
$\mathrm{COP}($ virtual $)=\alpha \times \mathrm{COP}$ (non-operated limb) $+\beta \times \mathrm{COP}$ (operated limb)

The third WBB sent a signal to the Wii console to control the game character. In this study, the sensitivity of the lower limb was determined through a preliminary study. The $\alpha$ value was set to 1.2 , and the $\beta$ value was set to 1 . The degree of the non-operative side reaction was more sensitive than that of the unilateral side, and so the degree amount of the operated leg was increased during the game. During the game training, the therapist always stood by to ensure safety. The CIMT game training was applied 3 times a week, 30 minutes a day, for 4 weeks [12]. The general game training group used the Slalom Ski program of the Nintendo Wii Fit game system as did the CIMT training group. Two WBBs were used in the same environment as the CIMT training group, but the same sensitivity was applied to both the surgical and non-surgical lower limbs without changing the sensitivity. The appropriate posture and the amount of each exercise to be performed were explained to the self-exercising group before proceeding the study, and received the instruction of the exercises so that they could exercise themselves from the week after the surgery. This exercise was revised and supplemented according to this study based on the self-exercises performed on the patients who had underwent knee arthroplasty [13]. During the 4-week period, the participants repeated 10 sets for each exercise and were recommended to exercise for 30 minutes, 3 times a week.

\section{Outcome measures}

The Western Ontario and McMaster Universities Osteoarthritis Index (WOMAC) is a tool used to assess the patients' level of pain, stiffness, and physical function. This questionnaire uses a Likert-type 5-point scale $(0=$ no, $1=$ weak, $2=$ moderate, and $3=$ severe). The correlation coefficient between the Korean version of the WOMAC was
0.79-0.89, and the Cronbach's alpha, which is the internal consistency, was 0.97 . The correlation coefficient between the patient's pain and general assessments was 0.30 , and the correlation coefficient with the overall physical function level was 0.41 [14].

The questionnaire method measures the fear of falling on a scale of 1 to 10 , which represents the subject's self-confidence level pertaining to performing certain activities. It is composed of 16 specific daily-life activities and calculates the average by adding the balance self-confidence scores. The score ranges from $0 \%$ to $100 \%$, and higher scores indicate having a greater amount of confidence in balance. The Cronbach's alpha of the Activity-specific Balance Confidence Scale was 0.99 and the test-retest reliability was $\mathrm{r}=0.82$, which is a reliable instrument showing a statistically significant relationship [15].

\section{Data and statistical analysis}

The IBM SPSS Statistics ver. 20.0 software (IBM Co., Armonk, NY, USA) was used for data analysis and normality test was performed with the Kolmogorov-Smirnov test to investigate the normal distribution of subject's general characteristics. The difference between pre- and post-intervention groups was investigated using the paired t-test. The one-way ANOVA was used for pre- and post-test differences, and the post-hoc test was performed by the Scheffe's method. The significance level was set at $p<0.05$.

\section{Results}

A total of 36 subjects participated in the study. The results were not statistically significant in age, height, surgery, and body weight among the three groups $(p>0.05)$. The general characteristics of the subjects are shown in Table 1. The changes in WOMAC scores are shown in Table 2. All 3

Table 1. General characteristics of subjects

$(\mathrm{N}=36)$

\begin{tabular}{lccrr}
\hline \multicolumn{1}{c}{ Variable } & $\begin{array}{c}\text { CIMT game training } \\
\text { group }(\mathrm{n}=12)\end{array}$ & $\begin{array}{c}\text { General game training } \\
\text { group }(\mathrm{n}=12)\end{array}$ & $\begin{array}{c}\text { Self-exercise } \\
\text { group }(\mathrm{n}=12)\end{array}$ & $\chi^{2} / \mathrm{F}$ \\
\hline Age $(\mathrm{y})$ & $69.58(3.94)$ & $70.17(6.18)$ & $73.58(6.71)$ & 1.703 \\
Height $(\mathrm{cm})$ & $153.78(8.75)$ & $151.27(5.35)$ & $151.63(8.13)$ & 0.388 \\
Weight $(\mathrm{kg})$ & $64.42(10.73)$ & $69.43(7.10)$ & $61.73(7.48)$ & 2.482 \\
Start date of training to postoperative (d) & $10.25(2.34)$ & $10.42(1.68)$ & $11.25(2.30)$ & 0.761 \\
Affected side (right/left) & $4 / 8$ & $7 / 5$ & $6 / 6$ & 1.560 \\
\hline
\end{tabular}

Values are presented as mean (SD) or number only.

CIMT: constraint-induced movement therapy. 
Table 2. Comparison of changes in pre and post-test of WOMAC among the three groups

$(\mathrm{N}=36)$

\begin{tabular}{|c|c|c|c|c|}
\hline Variable & $\begin{array}{l}\text { CIMT game training } \\
\text { group }(n=12)\end{array}$ & $\begin{array}{l}\text { General game training } \\
\text { group }(n=12)\end{array}$ & $\begin{array}{l}\text { Self-exercise } \\
\text { group }(n=12)\end{array}$ & $\mathrm{F}$ \\
\hline \multicolumn{5}{|l|}{ Pain (score) } \\
\hline Pre & $18.75(0.97)$ & $18.33(1.56)$ & $19.42(0.99)$ & 2.472 \\
\hline Post & $8.83(4.11)$ & $10.17(3.43)$ & $8.42(2.35)$ & 0.880 \\
\hline $\mathrm{t}$ & $8.161^{\mathrm{a}}$ & $8.720^{\mathrm{a}}$ & $18.241^{\mathrm{a}}$ & \\
\hline Change & $9.92(4.21)$ & $8.17(3.24)$ & $11.00(2.09)$ & 2.257 \\
\hline \multicolumn{5}{|l|}{ Stiffness (score) } \\
\hline Pre & $7.25(0.97)$ & $7.08(1.17)$ & $7.00(1.21)$ & 0.156 \\
\hline Post & $3.50(1.45)$ & $3.25(1.29)$ & $4.92(1.08)$ & $5.906^{\mathrm{a}}$ \\
\hline $\mathrm{t}$ & $8.409^{\mathrm{a}}$ & $6.820^{\mathrm{a}}$ & $8.016^{\mathrm{a}}$ & \\
\hline Change & $3.75(1.55)^{\mathrm{c}}$ & $3.83(1.95)^{\mathrm{c}}$ & $2.08(0.90)$ & 5.023 \\
\hline \multicolumn{5}{|c|}{ Physical function (score) } \\
\hline Pre & $59.67(9.66)$ & $58.75(9.13)$ & $58.83(8.59)$ & 0.037 \\
\hline Post & $19.67(6.18)$ & $30.83(11.01)$ & $46.50(8.86)$ & $27.490^{\mathrm{a}}$ \\
\hline $\mathrm{t}$ & $10.820^{\mathrm{a}}$ & $7.750^{\mathrm{a}}$ & $4.946^{\mathrm{a}}$ & \\
\hline Change & $40.00(12.81)^{\mathrm{b}, \mathrm{c}}$ & $27.92(12.47)^{\mathrm{c}}$ & $12.33(8.64)$ & 17.572 \\
\hline \multicolumn{5}{|l|}{ Total (score) } \\
\hline Pre & $85.67(9.58)$ & $84.17(9.56)$ & $85.25(9.27)$ & 0.080 \\
\hline Post & $32.00(6.55)$ & $44.25(12.76)$ & $59.83(9.89)$ & $23.087^{\mathrm{a}}$ \\
\hline $\mathrm{t}$ & $16.155^{\mathrm{a}}$ & $9.736^{\mathrm{a}}$ & $11.030^{\mathrm{a}}$ & \\
\hline Change & $53.67(11.51)^{\mathrm{b}, \mathrm{c}}$ & $39.92(14.20)^{\mathrm{c}}$ & $25.42(7.98)$ & 18.057 \\
\hline
\end{tabular}

Values are presented as mean (SD).

WOMAC: Western Ontario and McMaster Universities Osteoarthritis Index, CIMT: constraint-induced movement therapy.

${ }^{a}$ Significant difference pre and post training. ${ }^{b}$ Significant difference with general game training group. ${ }^{c}$ Significant difference with self-exercise group.

Table 3. Comparison of changes in pre and post-test on ABC Scale scores among the three groups

$(\mathrm{N}=36)$

\begin{tabular}{lcccc}
\hline ABC & $\begin{array}{c}\text { CIMT game training } \\
\text { group }(\mathrm{n}=12)\end{array}$ & $\begin{array}{c}\text { General game training } \\
\text { group }(\mathrm{n}=12)\end{array}$ & $\begin{array}{l}\text { Self-exercise } \\
\text { group }(\mathrm{n}=12)\end{array}$ & $\mathrm{F}$ \\
\hline Pre & $5.05(3.76)$ & $4.95(1.50)$ & $4.95(2.94)$ & 0.005 \\
Post & $53.18(8.62)$ & $36.25(18.18)$ & $36.51(18.48)$ & $4.543^{\mathrm{a}}$ \\
$\mathrm{t}$ & $-18.834^{\mathrm{a}}$ & $-6.426^{\mathrm{a}}$ & $-5.770^{\mathrm{a}}$ & $31.56(18.95)$ \\
Change & $48.13(8.95)^{\mathrm{b}, \mathrm{c}}$ & $31.30(16.88)^{\mathrm{b}}$ & & $4.620^{\mathrm{a}}$ \\
\hline
\end{tabular}

Values are presented as mean $(\mathrm{SD})$.

ABC: Activities-specific Balance Confidence, CIMT: constraint-induced movement therapy.

${ }^{a}$ Significant difference pre and post training. ${ }^{b}$ Significant difference with general game training group. ${ }^{\text {c }}$ Significant difference with self-exercise group.

groups showed significant improvement in pain, stiffness, and physical function between baseline and post-intervention values $(p<0.05)$. However, there were significantly greater improvements in stiffness in the CIMT game training group versus the general game training group $(p<0.05)$, which showed a larger decrease $(p<0.05)$. The overall score also showed a significant decrease in the CIMT game training group compared to the general game training and self-exercising groups $(p<0.05)$. All 3 groups showed significant improvements in ABC scores $(p<0.05)$. As a result of the post-hoc test for the $\mathrm{ABC}$, the CIMT game training group showed significantly greater improvements than the general game training and self-exercise group (Table 3).

\section{Discussion}

The major findings of this study showed that the WOMAC physical function and self-confidence in balance after CIMT game training for rehabilitation after total knee arthroplasty were significantly different compared to the two groups. 
Treatment with CIMT, which is commonly used for patients with nervous system impairments, especially stroke hemiplegia, is combined with games that provide balance training [12]. CIMT to the lower extremities decrease the frequency of use and increases the weight put onto the lower extremities. The purpose of this study was to achieve the same as effects observed using CIMT in orthopedic patients, which is widely used for upper extremity rehabilitation, in persons with stroke. In general, when applying CIMT to the upper extremity for a stroke patient, tools such as a sling or a glove are used to suppress movement. However, since it is difficult to use this tool on the legs to maintain postural stability and since it may interfere with movement, CIMT should not be applied to the lower extremities [16,17].

Pain after knee arthroplasty is closely related to the passage of time. In the early days, pain in present in almost all patients but it tends to disappear with the passage of time [18]. In addition, the patient's age, height, weight, and body mass index may be risk factors for infection, malnutrition, steroid use, long hospital care and diabetes, rheumatoid arthritis and psoriasis [19,20]. However, the personality and psychological state of the patient is also very important. In the present study, there was a significant difference in the amount of pre- and post-intervention pain between the groups over time, but no significant difference was found between groups. In this study, too, the center of mass was shifted during balance training using the Slalom Ski program. Because the legs must be used safely and effectively when moving the center of mass of the body, muscles are constantly used for the extension moment of the hips, knees and ankles, which assist to increase the strength of the extension, and furthermore.

The lower functional level was assessed using the WOMAC. There was a significant difference between preand post-intervention, but there was a significant difference in stiffness and physical function, except for pain, in comparison of between the three groups. Similar previous studies have shown that balance adjustment exercises and posture strategies improve knee joint function in patients with knee arthritis. This increased the control of neuromuscular feedback. This mechanism promoted dynamic balance, helped to improve physical performance as well as the function of the lower limbs [21]. This lowered the risk of injury and positively affected the balance responsiveness [22]. Therefore, it is similar to this study in which balance exercises performed after a knee artificial joint surgery positively influences the function of the hip. Another previous study showed a significant effect of various balance interventions on the functional outcome of the lower extremities. This activates both static and dynamic stabilization in the presence of a foam or balance board intervention. This helps to stimulate native receptors in the hips and ankles and improves the compensatory movement of the haptic sensation in the injured knee joint. This result indicates a meaningful improvement in lower limb function.

Changes in self-confidence levels on balance were assessed. As a result, all three groups showed significant changes preand post-intervention, and there was also a significant difference between the three groups. This is similar to the previous study in which virtual reality-based exercises analyzed the body and psychology associated with a patient's fall [23]. Also, in the correlation between balance self- confidence levels, function, and activities of daily living in TKR patients, participants with larger balance self-confidence levels showed improved functional performance and less difficulty in their activities of daily living [24]. This is because the patient was able to see the state of his or her balance and movement while performing the motion of the virtual reality as well as the stimulation from the vestibule system and proprioception due to the action of the visual and auditory feedback, which can control balance and reduce fear [25]. The increase in self-confidence in balance and the improvement of physical balance were also reported. In addition, game-based exercise training affected the physical and psychological functioning of the elderly.

The limitation of this study was that a follow-up was not performed because the patient was discharged within 4-5 weeks after the operation, and the study was conducted at a single hospital. Future research is expected to continue with follow-up studies, such as diversification of subjects, including games with various difficulties, and multiple game programs. The game-based CIMT program can enhance the interest levels and exercise ability. Therefore, in this study, intervention based on forced CIMT led to active participation of patients. The improvement in knee extension power and weight movement training effectively led to the improvement of static balance and dynamic balance abilities of the patients as well as the balance self-confidence levels.

\section{Acknowledgements}

This work was supported by the National Research Foundation of Korea (NRF) grant funded by the Korea government (MSIP) (No. 2016R1C1B2010420). 


\section{Conflict of Interest}

The authors declared no potential conflicts of interest with respect to the authorship and/or publication of this article.

\section{References}

1. Doma K, Grant A, Morris J. The effects of balance training on balance performance and functional outcome measures following total knee arthroplasty: a systematic review and meta-analysis. Sports Med 2018;48:2367-85.

2. Yoshida Y, Zeni J, Snyder-Mackler L. Do patients achieve normal gait patterns 3 years after total knee arthroplasty? J Orthop Sports Phys Ther 2012;42:1039-49.

3. Beswick AD, Wylde V, Gooberman-Hill R, Blom A, Dieppe P. What proportion of patients report long-term pain after total hip or knee replacement for osteoarthritis? A systematic review of prospective studies in unselected patients. BMJ Open 2012;2: e000435.

4. Harato K, Nagura T, Matsumoto H, Otani T, Toyama Y, Suda Y. Extension limitation in standing affects weight-bearing asymmetry after unilateral total knee arthroplasty. J Arthroplasty 2010; 25:225-9.

5. Gage WH, Frank JS, Prentice SD, Stevenson P. Postural responses following a rotational support surface perturbation, following knee joint replacement: frontal plane rotations. Gait Posture 2008;27:286-93.

6. Graves L, Stratton G, Ridgers ND, Cable NT. Energy expenditure in adolescents playing new generation computer games. $\mathrm{Br}$ J Sports Med 2008;42:592-4.

7. Nitz JC, Kuys S, Isles R, Fu S. Is the Wii Fit a new-generation tool for improving balance, health and well-being? A pilot study. Climacteric 2010;13:487-91.

8. Fung V, Ho A, Shaffer J, Chung E, Gomez M. Use of Nintendo Wii $\mathrm{Fit}^{\mathrm{TM}}$ in the rehabilitation of outpatients following total knee replacement: a preliminary randomised controlled trial. Physiotherapy 2012;98:183-8.

9. Christiansen CL, Bade MJ, Davidson BS, Dayton MR, StevensLapsley JE. Effects of weight-bearing biofeedback training on functional movement patterns following total knee arthroplasty: a randomized controlled trial. J Orthop Sports Phys Ther 2015; 45:647-55.

10. Abbas C, Daher J. Pilot study: post-operative rehabilitation pathway changes and implementation of functional closed kinetic chain exercise in total hip and total knee replacement patient. J Bodyw Mov Ther 2017;21:823-9.

11. Levinger P, Zeina D, Teshome AK, Skinner E, Begg R, Abbott $\mathrm{JH}$. A real time biofeedback using Kinect and Wii to improve gait for post-total knee replacement rehabilitation: a case study report. Disabil Rehabil Assist Technol 2016;11:251-62.
12. Choi HS, Shin WS, Bang DH, Choi SJ. Effects of game-based constraint-induced movement therapy on balance in patients with stroke: a single-blind randomized controlled trial. Am J Phys Med Rehabil 2017;96:184-90.

13. Negus JJ, Cawthorne DP, Chen JS, Scholes CJ, Parker DA, March LM. Patient outcomes using Wii-enhanced rehabilitation after total knee replacement - the TKR-POWER study. Contemp Clin Trials 2015;40:47-53.

14. Bae SC, Lee HS, Yun HR, Kim TH, Yoo DH, Kim SY. Cross-cultural adaptation and validation of Korean Western Ontario and McMaster Universities (WOMAC) and Lequesne osteoarthritis indices for clinical research. Osteoarthritis Cartilage 2001;9: 746-50.

15. Powell LE, Myers AM. The Activities-specific Balance Confidence (ABC) Scale. J Gerontol A Biol Sci Med Sci 1995;50A: M28-34.

16. Ding Q, Stevenson IH, Wang N, Li W, Sun Y, Wang Q, et al. Motion games improve balance control in stroke survivors: a preliminary study based on the principle of constraint-induced movement therapy. Disp 2013;34:125-31.

17. Numata K, Murayama T, Takasugi J, Oga M. Effect of modified constraint-induced movement therapy on lower extremity hemiplegia due to a higher-motor area lesion. Brain Inj 2008;22:898904.

18. Cho WS, Ahn HS, Kim MY, Seol ES, Lee SW, Choi JW. Pain after total knee arthroplasty. J Korean Orthop Assoc 2006;41:12933.

19. Mahomed NN, Liang MH, Cook EF, Daltroy LH, Fortin PR, Fossel AH, et al. The importance of patient expectations in predicting functional outcomes after total joint arthroplasty. J Rheumatol 2002;29:1273-9.

20. Wilson MG, Kelley K, Thornhill TS. Infection as a complication of total knee-replacement arthroplasty. Risk factors and treatment in sixty-seven cases. J Bone Joint Surg Am 1990;72:87883.

21. Gauchard GC, Vançon G, Meyer P, Mainard D, Perrin PP. On the role of knee joint in balance control and postural strategies: effects of total knee replacement in elderly subjects with knee osteoarthritis. Gait Posture 2010;32:155-60.

22. Liao CD, Liou TH, Huang YY, Huang YC. Effects of balance training on functional outcome after total knee replacement in patients with knee osteoarthritis: a randomized controlled trial. Clin Rehabil 2013;27:697-709.

23. Jung DI, Seo TH, Ko DS. Comparative analysis of fall-related physicopsychological according to virtual exercise and lumbar stabilization exercise in the patient with stroke. Int J Content 2012;12:274-82.

24. Webster KE, Feller JA, Wittwer JE. Balance confidence and function after knee-replacement surgery. J Aging Phys Act 2006; 14:181-91.

25. Roh KH. The effect of rhythmic dance movement training on the gait length, dynamic valance, depression, quality of life. Korean J Rehabil Nurs 2003;6:70-8. 